\title{
Uso del láser terapéutico en el control del dolor en ortodoncia
}

\author{
Use of laser therapy in pain control in orthodontics
}

\author{
Holmberg Peters F', Zaror Sánchez $C^{2}$, Fabres Suarez R³, Sandoval Vidal P4
}

\section{RESUMEN}

Objetivo: Determinar si el láser de baja frecuencia (LLLT) es efectivo en disminuir la percepción del dolor posterior a la aplicación de separaciones molares para ortodoncia. Material y Método: Se realizó un ensayo clínico aleatorizado con enmascaramiento simple en 30 alumnos entre 16 y 20 años, a quienes se les aplicó separaciones elásticas entre los molares permanentes. Los voluntarios fueron designados en dos grupos: 15 pacientes en el grupo experimental quienes fueron tratados con LLLT (Photon Lase, DMC USA Corp.) por 30 segundos en cada molar permanente derecho y 15 pacientes en el grupo control quienes recibieron como placebo la colocación del láser inactivo con el mismo protocolo empleado en el grupo experimental. En total 30 dientes fueron incluidos en cada grupo. La percepción del dolor fue evaluada a las 3, 12, 24 horas y a los días 2, 3, 4, 5 y 7 posterior a la aplicación de LLLT a través de un cuestionario estándar de autollenado haciendo uso de la escala visual análoga del dolor (EVA). Resultados: El $61.6 \%$ de los pacientes del grupo de control presentó algún grado de dolor durante el periodo de seguimiento en comparación del $38.64 \%$ del grupo experimental $(p=0.004)$. Si bien la intensidad del dolor alcanzó su punto máximo a las 12 horas las diferencias de los promedios entre ambos grupos también fue estadísticamente significativa $(<0.001)$. Conclusión: EI LLLT demostró ser una herramienta útil en el control del dolor posterior a la aplicación de separaciones molares.

Rev. Clin. Periodoncia Implantol. Rehabil. Oral Vol. 4(3); 114-116, 2011.

Palabras clave: Dolor, tratamiento ortodoncia, láser terapéutico.

\section{ABSTRACT}

Objective: To determine the effectiveness of low-level laser therapy (LLLT) to decrease the perception of pain after application of orthodontic molar separations. Material and Methods: We performed a randomized single-blind in 30 students between 16 and 20 years who were administered elastic separation between the permanent molars. Volunteers were assigned into two groups: 15 patients in the experimental group who were treated with LLLT (Photon Lase, DMC USA Corp.) for 30 seconds on each permanent right molar and 15 patients in the control group who received placebo-like placement inactive laser with the same protocol used in the experimental group. Altogether 30 teeth were included in each group. Pain perception was assessed at 3, 12, 24 hours and 2, 3, 4, 5 and 7 after application of LLLT through a standard questionnaire using the visual analogue scale of pain (EVA). Results: $61.6 \%$ of patients in the control group had some degree of pain during the follow-up period in comparison with the $38.64 \%$ of the experimental group $(p=0.004)$. Although pain intensity peaked at 12 hours the mean differences between groups was statistically significant $(<0.001)$. Conclusion: LLLT proved to be a useful tool in controlling pain after the application of separation molars.

Rev. Clin. Periodoncia Implantol. Rehabil. Oral Vol. 4(3); 114-116, 2011.

Key words: Pain, orthodontic treatment, low-level laser therapy.

\section{INTRODUCCIÓN}

La utilización del láser en odontología ha tenido una constante evolución y desarrollo; cada vez son más las especialidades odontológicas en las que se aplican las diferentes variedades de láser, ya sea en procesos diagnósticos o terapéuticos. En la especialidad de ortodoncia puede ser de gran utilidad en el control del dolor y como acelerador de los procesos regenerativos y de cicatrización tisular ${ }^{(1,2)}$.

Se encuentra ampliamente descrito que posterior a la aplicación de fuerzas ortodóncicas se produce un periodo de disconfort o dolor inicial que dura de 2 a 4 días $^{(3-5)}$. Desde el punto de vista histológico, este fenómeno se explicaría por la mayor compresión de las fibras periodontales con mayor daño tisular y la mayor repuesta dolorosa(6).

La asociación de dolor con ortodoncia es una causa de rechazo al tratamiento en algunos pacientes, a su vez el uso de analgesia durante el tratamiento esta cuestionado por algunos autores ya que interferiría con el movimiento dentario justificándose la utilización de otros recursos en el control y manejo del dolor ${ }^{(7)}$. Es así como algunos autores han aprovechado las propiedades analgésicas del láser de baja frecuencia para disminuir el dolor tras los ajustes ortodóncicos ${ }^{(8-10)}$. Este es el caso del estudio publicado por Lim y cols. en $1995^{(11)}$, donde los resultados demuestran un menor nivel de dolor en la escala analógica visual comparando con el grupo control, pero sin encontrar diferencias estadísticamente significativas.

El objetivo de este estudio es evaluar la efectividad del láser terapéutico en el control del dolor en pacientes de ortodoncia posterior a la aplicación de separaciones molares.

\section{MATERIAL Y MÉTODO}

Participantes

Se realizó un ensayo clínico aleatorizado en 30 alumnos entre 16 y 20 años del Liceo Municipal González Vásquez de la ciudad de Nueva Imperial, IX Región, Chile, para determinar si el láser de baja frecuencia es efectivo en disminuir la percepción del dolor posterior a la aplicación de separaciones molares en ortodoncia.

Se excluyeron aquellos individuos que padecían alguna enfermedad sistémica de base, alteraciones neurológicas, problemas siquiátricos y/o que hayan consumido analgésicos durante la última semana.

1. Magíster@ en Odontología. Especialista en Periodoncia. Especialista en Ortodoncia y Ortopedia DentoMaxiloFacial. Universidad de la Frontera. Temuco, Chile. 2. Magíster@ en Epidemiología Clínica. Especialista en Odontopediatría. Docente del Programa de Especialización en Odontopediatría. Universidad de la Frontera. Temuco, Chile.

3. Cirujano Dentista. Docente Pregrado. Universidad de la Frontera. Temuco, Chile.

4. Magíster en Educación. Especialista en Ortodoncia y Ortopedia DentoMaxiloFacial. Jefe Programa de Especialización en Ortodoncia y Ortopedia DentoMaxiloFacial. Universidad de La Frontera. Temuco, Chile.

Correspondencia autor: Fernando Holmberg Peters. fernandoholmberg@yahoo.com. Manuel Mont\#112, $4^{\circ}$ piso. Dpto. Odontología Integral of. 420, Facultad de Medicina, Universidad de la Frontera. Temuco, Chile. Trabajo recibido el 02/08/2011. Aprobado para su publicación el 14/10/2011. Financiamiento: Trabajo inserto en el Programa de Magíster en Odontología de la Universidad de La Frontera. 
Muestra

Según la literatura se espera un porcentaje de alrededor de un $75 \%$ de pacientes que presenten sintomatología dolorosa posterior a la instalación de aparatos de ortodoncia fija. El cálculo de tamaño de muestra se hizo para detectar diferencias de por lo menos 40 puntos porcentuales en las proporciones sobre la percepción del dolor a favor de los pacientes que reciben terapia de láser. Lo anterior, sumado a un nivel de significación de $5 \%$ y un poder estadístico de $80 \%$, nos permite estimar que el tamaño de muestra mínimo suficiente es de 28 piezas por grupo, la que fue usada como unidad de medida.

\section{Aleatorización}

Los individuos con consentimiento informado firmado fueron aleatoriamente asignados a uno de los 2 grupos, mediante una designación numérica aleatoria hecha por computador. La asignación fue ocultada en un sobre cerrado hasta el inicio del tratamiento para el clínico a cargo de la intervención.

A través de la aleatorización los voluntarios fueron designados en dos grupos: 15 pacientes en el grupo 1 donde fueron tratados con LLLT (Photon Lase, DMC USA Corp.) y 15 pacientes en el grupo 2 quienes recibieron como placebo la colocación del láser inactivo. En total 30 dientes fueron incluidos en cada grupo (primeros molares superiores e inferiores derechos).

\section{Descripción de la Intervención}

A todos los pacientes se les aplicó separaciones elásticas (X-Ring Separators, American Orthodontics, USA) entre los todos los molares permanentes en estudio, por mesial y distal.

Los pacientes asignados al grupo 1 recibieron una aplicación única de LLLT (Photon Lase, DMC USA Corp., Longitud de onda: 660$685 \mathrm{~nm}$ : Emisor visible; Longitud de onda: 790-830 nm: Emisor invisible; Potencia útil de láser rojo e infrarrojo: $100 \mathrm{~mW}$ ) por 30 segundos (15 segundos por vestibular y 15 segundos por palatino/lingual) en cada molar permanente derecho (1.6 y 4.6), a una distancia de 5 a $8 \mathrm{~mm}$ y en ángulo recto con la mucosa.

A los pacientes del grupo 2 se les aplicó el LLLT sin irradiación activa del láser, por lo que el paciente se encontraba enmascarado al tratamiento.

La percepción del dolor fue evaluada a las 3,12 y 24 horas y a los días 2, 3, 4, 5 y 7 posterior al tratamiento a través de un cuestionario estándar de autoreporte haciendo uso de la escala visual análoga del dolor (EVA). La EVA consiste en una línea recta de $10 \mathrm{~cm}$ de longitud, con leyendas "SIN DOLOR" y "DOLOR MÁXIMO" en cada extremo(12). Los sujetos fueron instruidos a marcar en la línea con una $X$ el grado de dolor que siente de acuerdo a su percepción individual, midiendo el dolor en centímetros desde el punto cero hasta la línea marcada por el sujeto.

Todos los pacientes fueron instruidos por un operador para completar la encuesta en el hogar durante los próximos 7 días y se les instruyó para que no tomen ningún analgésico. En el caso que esto ocurriese debían registrar el día y hora del consumo del medicamento. El paciente que consuma medicamentos no será consignado en el análisis.

Sus informes fueron recogidos por un investigador ajeno a la etapa de tratamiento.

\section{Análisis Estadístico}

Los análisis estadísticos se llevaron a cabo con el programa STATA 10 (StataCorp LP, USA). Diferencias significativas en la prevalencia del dolor se evaluaron con la prueba exacta de Fisher con un nivel de significación de un 0.05 . Los datos de estos informes se compararon mediante análisis de la varianza (ANOVA). Cuando las diferencias entre los tratamientos se encontraron, la prueba de Bonferroni para comparaciones múltiples se aplicó. Las pruebas se realizaron al nivel del $5 \%$ de significación.

\section{RESULTADOS}

La muestra fue constituida por 30 individuos entre 16 y 20 años con una media de $18+0.86$ años. De los cuales 25 eran mujeres y 5 eran hombres. Los pacientes del grupo 1 estaba compuesta por 12 mujeres y 3 hombres con un promedio de edad 17 años y el grupo 2 estaba compuesta por 13 mujeres y 2 hombres con un promedio de edad 16.6 años.

El $61.6 \%$ de los pacientes del grupo de control presentó algún grado de dolor durante el periodo de seguimiento en comparación del $38.64 \%$ del grupo experimental, diferencia que fue estadísticamente significativa $(p=0.004)$.

La Tabla 1 muestra la prevalencia de dolor en el tiempo por cada grupo de estudio. La prevalencia de dolor en el grupo 1 a las 3 horas es de un $50 \%$ y el grupo 2 fue de un $73.3 \%$, diferencia que no fue estadísticamente significativa (0.063). En el segundo control (12 horas) la prevalencia de dolor fue de un $40 \%$ en el grupo 1 y de un $80 \%$ en el grupo 2 diferencia que fue significativa entre ambos grupos (0.002). Al final del seguimiento la prevalencia de dolor en el grupo 1 fue de $6.67 \%$ en relación a la del grupo 2 que fue de $63.3 \%$ diferencia que fue estadísticamente significativa $(<0.001)$.

Tabla 1. Porcentaje de individuos con presencia de dolor en los diferentes períodos.

\begin{tabular}{|l|c|c|c|}
\hline Periodo & $\begin{array}{c}\text { Láser } \\
(\mathbf{\%})\end{array}$ & $\begin{array}{c}\text { Control } \\
(\mathbf{\%})\end{array}$ & $\mathbf{p}^{*}$ \\
\hline 3 horas & 50.00 & 73.33 & 0.063 \\
\hline 12 horas & 40.00 & 80.00 & $0.002^{*}$ \\
\hline 24 horas & 33.33 & 73.33 & $0.002^{*}$ \\
\hline Día 2 & 16.67 & 76.67 & $<0.001^{*}$ \\
\hline Día 3 & 23.33 & 73.33 & $<0.001^{*}$ \\
\hline Día 4 & 13.33 & 73.33 & $<0.001^{*}$ \\
\hline Día 5 & 6.67 & 70.00 & $<0.001^{*}$ \\
\hline Día 7 & 6.67 & 63.33 & $<0.001^{*}$ \\
\hline
\end{tabular}

${ }^{*}$ Diferencia estadisticamente significativa test exacto de Fisher $(p<0.05)$.

La Tabla 2 muestra la media de intensidad del dolor por grupo de estudio durante el periodo de seguimiento. A las 3 horas la media de intensidad dolor según EVA fue de $0.43+1.33$ en el grupo 1 y de $2.3+1.88$ en el grupo 2 ( $p=0.0019)$ y al quinto día la intensidad promedio de dolor fue de $0.07+0.25$ en el grupo 1 y de $1.6+1.43$ en el grupo 2 , valores que se repiten al séptimo día. Si bien la intensidad del dolor alcanzó su punto máximo a las 12 horas las diferencias de los promedios entre ambos grupos también fue estadísticamente significativa $(<0.001)$.

Ningún paciente reportó consumo de analgésicos durante el periodo de seguimiento.

Tabla 2. Intensidad de dolor en los diferentes períodos.

\begin{tabular}{|l|c|c|c|}
\hline Periodo & $\begin{array}{c}\text { Láser } \\
\text { (media } \pm \text { DE) }\end{array}$ & $\begin{array}{c}\text { Control } \\
\text { (media } \pm \text { DE) }\end{array}$ & $\mathbf{p}^{*}$ \\
\hline 3 horas & $0.43 \pm 1.33$ & $2.3 \pm 1.88$ & 0.0019 \\
\hline 12 horas & $0.57 \pm 0.77$ & $2.27 \pm 1.74$ & $<0.001$ \\
\hline 24 horas & $0.4 \pm 0.62$ & $2.23 \pm 1.81$ & $<0.001$ \\
\hline Día 2 & $0.23 \pm 0.57$ & $2.17 \pm 1.66$ & $<0.001$ \\
\hline Día 3 & $0.23 \pm 0.43$ & $1.6 \pm 1.35$ & $<0.001$ \\
\hline Día 4 & $0.13 \pm 0.35$ & $1.57 \pm 1.3$ & $<0.001$ \\
\hline Día 5 & $0.07 \pm 0.25$ & $1.6 \pm 1.43$ & $<0.001$ \\
\hline Día 7 & $0.07 \pm 0.25$ & $1.4 \pm 1.45$ & $<0.001$ \\
\hline
\end{tabular}

${ }^{*}$ Diferencia estadísticamente significativa $(p<0.05)$

\section{DISCUSIÓN}

EL LLLT fue elegido en este estudio por sobre otros tipos de láser, basado en estudios previos publicados en la literatura, los cuales han demostrado que la penetración del LLLT dentro de los tejidos es más efectivo que el láser visible frecuentemente usado en tratamientos de encías y piel $(8,13,14)$.

EL tiempo de aplicación del láser en este estudio fue basado en los reportes de Turhani y cols. en el 2006, quien determinó que una mínima aplicación de LLLT es eficaz en la reducción del dolor.

El uso de LLLT para el control del dolor en pacientes de ortodoncia ha sido reportado previamente(8-11,13-16). Sin embargo, los resultados no han sido estadísticamente significativos o se sugiere más investigaciones respecto al tema. Nuestro ensayo clínico demostró que LLLT es efectivo en el control del dolor asociado a las aplicaciones de separaciones molares en pacientes en tratamiento de ortodoncia $(p=0.004)$. 
La percepción del dolor en diferentes edades es controversial en la literatura ${ }^{(17-19)}$. Nuestro estudio se limitó a adolecentes y adultos jóvenes con un promedio de edad de 18 años donde ha demostrado ser altamente efectivo. Obviamente nuestros resultados no se pueden extrapolar a pacientes preadolescentes, quienes son frecuentemente sometidos a tratamientos de ortodoncia.

Según Furstman ${ }^{(6)}$ el dolor tiende a aparecer aproximadamente 2 horas después de la instalación de aparatos de ortodoncia. Por otro lado $\mathrm{Ngan}^{(4)}$ determinó que la percepción del dolor fue más pronunciada en los primeros 3 días después de la instalación de los aparatos de ortodoncia, alcanzando su intensidad máxima entre las 24 y 48 horas después de la inserción de separadores molares. Estudios previos han mostrado que los pacientes se acostumbran al dolor dentro de los primeros 3 a 7 días de la instalación de los aparatos de ortodoncia ${ }^{(3,4,17)}$. Todos estos acontecimientos concuerdan con nuestros resultados, que mostraron que la intensidad del dolor alcanzó su punto máximo a las 12 horas después de la aplicación de separaciones molares, comenzando a disminuir a las 48 horas y estabilizándose al quinto día.

La principal limitación de este estudio tiene que ver con la dificultad en la medición del dolor, dada su naturaleza subjetiva y la variabilidad individual en el umbral y sensibilidad de éste. Se decidió usar la escala visual análoga porque ha demostrado ser un método confiable en la medición del dolor y porque la mayoría de los estudios que han asociado dolor con aparatología ortodóntica han sido conducidos usando la $\operatorname{EVA}^{(4,12,20)}$

El uso de analgésico es la terapia de elección para la reducción del dolor en pacientes sometidos a tratamientos de ortodoncia, sin embargo, su uso se ha visto cuestionado en el último tiempo dado sus efectos colaterales, su contraindicación en pacientes alérgicos y su interferencia en el movimiento dentario dado por algunos antiinflamatorios no esteroidales ${ }^{(21,22)}$. Dentro de este contexto el LLLT parece ser una alternativa válida en el manejo del dolor de pacientes sometidos a tratamiento de ortodoncia al ser una terapia no invasiva, de fácil aplicación y sin efectos adversos sobre los tejidos. La única contraindicación que encontramos al uso del LLLT en el control del dolor en ortodoncia tiene que ver con el tiempo total de aplicación en ambas arcadas que podría alcanzar los 30 minutos, lo que concuerda con otros estudios $^{(9,11,13,14)}$

A la luz de la evidencia presentada surge la necesidad de realizar ensayos clínicos aleatorizados que comparen estos tratamientos y estudios de costo-efectividad que justifiquen el uso del LLLT por sobre los analgésicos en el manejo del dolor en ortodoncia.

En conclusión, la aplicación de láser terapéutico de baja frecuencia (LLLT) es un método eficiente para controlar el dolor en los pacientes de ortodoncia. Los pacientes irradiados con LLLT que manifiestan dolor al aplicarles separaciones molares son significativamente menores comparados con el grupo control y esto es mantenido hasta séptimo día. La intensidad del dolor percibida por estos individuos es significativamente menor que la percibida en los controles.

\section{AGRADECIMIENTOS} frecuencia.

A Biofotónica Chile, empresa que facilitó el láser de baja Al Director del establecimiento educacional que permitió el ingreso a los investigadores.

\section{CONFLICTOS DE INTERÉS}

Los autores declaran no tener conflictos de interés.

\section{REFERENCIAS BIBLIOGRÁFICAS}

1. Walsh LJ. The current status of low level laser therapy in dentistry. Part 1. Soft tissue applications. Aust Dent J, 1997 Aug; 42(4): 247-254. 2. Walsh LJ. The current status of low level laser therapy in dentistry. Part 2. Hard tissue applications. Aust Dent J, 1997 Oct; 42(5): 302-306.

3. Jones ML. An investigation into the initial discomfort caused by placement of an archwire. Eur J Orthod, 1984; 6: 48-54.

4. Ngan P, Kess B, Wilson S. Perception of discomfort by patients undergoing orthodontic treatment. Am J Orthod Dentofacial Orthop, 1989 Jul; 96(1): 47-53.

5. Soltis JE, Nalfoor PR, Bowman DC. Changes in ability ofpatients to differentiate intensity of forces applied to maxillary central incisors during orthodontic treatment. J Dent Res, 1971; 50(3): 590-596.

6. Furstman L, Bernick S. Clinical considerations of the periodontium. Am J Orthod, 1972 Feb; 61(2): 138-155.

7. Ohshiro T, Calderhead RG. Development of low reactive-level laser therapy and its present status. J Clin Laser Med Surg, 1991 Aug; 9(4): 267-275.

8. Harazaki M, Isshiki Y. Soft laser irradiation effects on pain reduction in orthodontic treatment. Bull Tokyo Dent Coll, 1997 Nov; 38(4): 291-295.

9. Tortamano A, Lenzi DC, Haddad AC, Bottino MC, Domínguez GC, Vigorito JW. Low-level laser therapy for pain caused by placement of the first orthodontic archwire: A randomized clinical trial. Am J Orthod Dentofacial Orthop, 2009 Nov; 136(5): 662-667.

10. Turhani D, Scheriau M, Kapral D, Benesch T, Jonke E, Bantleon HP. Pain relief by single low-level laser irradiation in orthodontic patients undergoing fixed appliance therapy. Am J Orthod Dentofacial Orthop, 2006 Sep; 130(3): 371-377.

11. Lim HM, Lew KK, Tay DK. A clinical investigation of the efficacy of low level laser therapy in reducing orthodontic postadjustment pain. Am J Orthod Dentofacial Orthop, 1995 Dec; 108(6): 614-622.

12. Price DD, McGrath PA, Rafii A, Buckingham $B$. The validation of visual analogue scales as ratio scale measures for chronic and experimental pain. Pain, 1983 Sep; 17(1): 45-56.
13. Harazaki $M$, Takahashi $H$, Ito $A$, Isshiki $Y$. Soft laser irradiation induced pain reduction in orthodontic treatment. Bull Tokyo Dent Coll, 1998 May; 39(2): 95-101.

14. Katoh H, Chen RC, Chai CT, Saito S, Yamasaki K, Shibasaki Y. Effect of far infrared rays radiation on pain reaction associated with orthondontic tooth movement. J Japan Orthod Soc, 1997; 56: 17-25.

15. Xiaoting L, Yin T, Yangxi C. Interventions for pain during fixed orthodontic appliance therapy. A systematic review. Angle Orthod, 2010 Sep; 80(5): 925-932.

16. Youssef M, Ashkar S, Hamade E, Gutknecht N, Lampert F, Mir M. The effect of low-level laser therapy during orthodontic movement: A preliminary study. Lasers Med Sci, 2008 Jan; 23(1): 27-33.

17. Scheurer PA, Firestone AR, Burgin WB. Perception of pain as a result of orthodontic treatment with fixed appliances. Eur J Orthod, 1996 Aug; 18(4): 349-357.

18. Wilson S, Ngan P, Kess B. Time course of the discomfort in young patients undergoing orthodontic treatment. Pediatr Dent, 1989 Jun; 11(2): 107-110.

19. Brown DF, Moerenhout RG. The pain experience and psychological adjustment to orthodontic treatment of preadolescents, adolescents, and adults. Am J Orthod Dentofacial Orthop, 1991 Oct; 100(4): 349-356.

20. Fujiyama K, Deguchi T, Murakami T, Fujii A, Kushima K, TakanoYamamoto T. Clinical effect of $\mathrm{CO}(2)$ laser in reducing pain in orthodontics. Angle Orthod, 2008 Mar; 78(2): 299-303.

21. El-Kinay WA. Pharmacological control of tooth movement. The combined effects of indometacin and prostaglandin El on orthodontic movement in dog. J Japan Orthod Soc, 1992; 51: 440-449.

22. Kyrkanides S, O'Banion MK, Subtelny JD. Nonsteroidal antiinflammatory drugs in orthodontic tooth movement: Metalloproteinase activity and collagen synthesis by endothelial cells. Am J Orthod Dentofacial Orthop, 2000 Aug; 118(2): 203-209. 